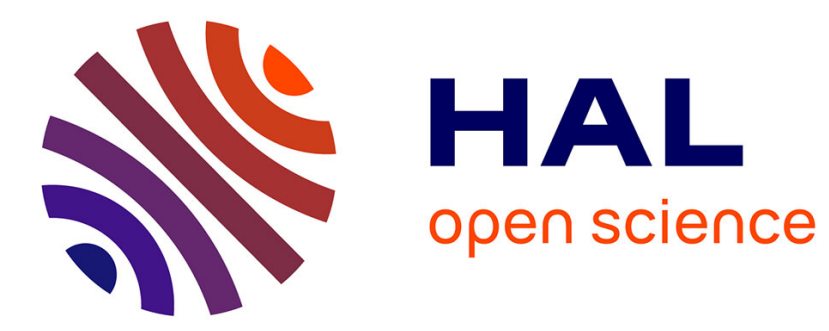

\title{
Resistive switching in silicon suboxide films
}

Adnan Mehonic, Sebastien Cueff, Maciej Wojdak, Stephen Hudziak, Olivier

Jambois, Christophe Labbe, Blas Garrido, Richard Rizk, Anthony J. Kenyon

\section{To cite this version:}

Adnan Mehonic, Sebastien Cueff, Maciej Wojdak, Stephen Hudziak, Olivier Jambois, et al.. Resistive switching in silicon suboxide films. Journal of Applied Geophysics, 2012, 111 (7), pp.074507. 10.1063/1.3701581 . hal-01148232v2

\section{HAL Id: hal-01148232 \\ https://hal.science/hal-01148232v2}

Submitted on 10 Dec 2015

HAL is a multi-disciplinary open access archive for the deposit and dissemination of scientific research documents, whether they are published or not. The documents may come from teaching and research institutions in France or abroad, or from public or private research centers.
L'archive ouverte pluridisciplinaire HAL, est destinée au dépôt et à la diffusion de documents scientifiques de niveau recherche, publiés ou non, émanant des établissements d'enseignement et de recherche français ou étrangers, des laboratoires publics ou privés. 


\section{Resistive switching in silicon sub-oxide films}

Adnan Mehonic ${ }^{1 *}$, Sébastien Cueff ${ }^{2}$, Maciej Wojdak ${ }^{1}$, Stephen Hudziak ${ }^{1}$, Olivier Jambois ${ }^{3}$, Christophe Labbé $^{2}$, Blas Garrido ${ }^{3}$ Richard Rizk ${ }^{2}$ and Anthony J. Kenyon ${ }^{1 *}$

${ }^{1}$ Department of Electronic \& Electrical Engineering, UCL, Torrington Place, London WC1E 7JE, UK ${ }^{2}$ CIMAP, UMR CNRS 6252 ENSICAEN, 6 Boulevard Maréchal Juin, 14050 Caen Cedex 4, France ${ }^{3}$ MIND-IN2UB, Dept. Electrònica, Universitat de Barcelona, Martí i Fanquès 1, 08028, Barcelona, CAT, Spain

*a.mehonic@ee.ucl.ac.uk; t.kenyon@ee.ucl.ac.uk 


\section{Abstract}

We report a study of resistive switching in a silicon-based memristor/resistive RAM (RRAM) device in which the active layer is silicon-rich silica. The resistive switching phenomenon is an intrinsic property of the silicon-rich oxide layer and does not depend on the diffusion of metallic ions to form conductive paths. In contrast to other work in the literature, switching occurs in ambient conditions, and is not limited to the surface of the active material.

We proposed a switching mechanism driven by competing field-driven formation and current-driven destruction of filamentary conductive pathways. We demonstrate that conduction is dominated by trap assisted tunnelling through non-continuous conduction paths consisting of silicon nanoinclusions in a highly non-stoichiometric suboxide phase. We hypothesise that such nanoinclusions nucleate preferentially at internal grain boundaries in nanostructured films.

Switching exhibits the pinched hysteresis I/V loop characteristic of memristive systems, and on/off resistance ratios of $10^{4}: 1$ or higher can be easily achieved. Scanning Tunnelling Microscopy suggests that switchable conductive pathways are 10nm in diameter or smaller. Programming currents can be as low as $2 \mu \mathrm{A}$, and transition times are on the nanosecond scale.

\section{Introduction}

Non-volatile memories based on resistive switching have recently attracted significant attention ${ }^{1}$. In particular, the two-terminal elements known as memristors ${ }^{2}$ offer a potential solution to the problem of decreased controllability of charge in semiconductor memories as devices scale to ever-smaller dimensions. First postulated by Leon Chua in in 1971 as the "missing" fourth passive circuit element ${ }^{2}$, the first practical realisation of the memristor, in titanium dioxide, was not reported until $2008^{3}$. Such devices have a wide range of potential applications in high-density memories, novel processor architectures, and neural networks. Consequently, the development of a practical CMOS device is a very attractive prospect for integration with current silicon technologies. Proof-of-principle memory systems exploiting memristors have already been demonstrated ${ }^{1,3-7}$, typically consisting of multilayer structures in which conductive pathways form under the application of external fields. Titanium 
dioxide multilayers, exploiting field-driven redistribution of oxygen ions, were the first reported memristor devices ${ }^{3}$, but there have also been promising reports of silicon-based resistive switches ${ }^{4,8}$. In the latter case, two approaches have been successful: field-driven diffusion of silver ions from metal contacts into amorphous silicon layers ${ }^{5,8}$ and switchable conductive pathways on the surface of silicon-rich silica $\left(\mathrm{SiO}_{\mathrm{x}}\right)$ pillars ${ }^{4}$. However, in CMOS processing the diffusion of metallic ions is undesirable, as this could potentially endanger the operation of surrounding electronics. The intrinsic resistive switching of silicon oxide is a more appealing mechanism. The first such system was demonstrated by Yao et $\mathrm{al}^{4}$, who reported a switchable silicon conductive path formed on the vertical surface a of silicon-rich silica pillar. However, the operation of such a device is possible only under vacuum due to oxidation of silicon conductive pathways on the device surface under ambient conditions. The authors reported no evidence of high-contrast controllable resistive switching in continuous films of silicon oxide: instead, silicon nanofilaments at vertical surfaces of mesa structures were proposed as the switching elements. In contrast, we demonstrate here a device operating under ambient conditions that relies on switching within a continuous thin film of silicon-rich silica. The device can be cycled between high resistance "OFF" and low resistance "ON" states with a resistance contrast of 10,000 or more. Both states are stable, persisting for at least 120 hours, and likely much longer

\section{Experimental details}

Our test devices (Figure 1(b)) contain thin (15-120nm) $\mathrm{SiO}_{\mathrm{x}}$ layers sandwiched between a p-type silicon wafer and n-type top electrodes (a range of electrode sizes was used - from $6 \mathrm{~mm} \times 4 \mathrm{~mm}$ to $125 \mu \mathrm{m} \times 125 \mu \mathrm{m})$. To address thermal budget issues during fabrication, we investigated two configurations: one with n-type polycrystalline silicon top electrodes, requiring a $950^{\circ} \mathrm{C}$ anneal to optimise conductivity, and a second with indium tin oxide (ITO) electrodes, deposited at room temperature. The former is readily integrated with front-end CMOS processes; the latter allows memristors to be integrated onto existing device layers without high temperature processing. The $\mathrm{SiO}_{\mathrm{x}}$ layers were deposited by magnetron co-sputtering onto p-type, B-doped silicon (100) wafers. Samples were deposited at $500^{\circ} \mathrm{C}$. Two confocal cathodes were used: $\mathrm{SiO}_{2}$ and $\mathrm{Si}$, under a 
pure argon plasma. Layer thicknesses between $15 \mathrm{~nm}$ and $120 \mathrm{~nm}$ (measured by spectroscopic ellipsometry) were used for different devices, and excess silicon content was between 11 and 30at\% for different samples (measured by XPS: Perkin-Elmer PHI-5500). Results reported here were from samples containing 11 at $\%$ excess Si. Samples were annealed at $900^{\circ} \mathrm{C}$ or at $500^{\circ} \mathrm{C}$ post-deposition in an argon atmosphere. The initial sample annealing temperature had little effect on the switching behaviour of the devices.

For one batch of samples, $185 \mathrm{~nm}$ of n-type silicon (phosphorous doped, resistivity $10 \mathrm{~m} \Omega \mathrm{cm}$ ) was deposited on top of the $\mathrm{SiO}_{\mathrm{x}}$ layer by Low Pressure Chemical Vapour Deposition (LPCVD). After growth, samples were annealed at $950^{\circ} \mathrm{C}$ for 30 minutes in nitrogen to activate the dopants and achieve a final resistivity of $1 \mathrm{~m} \Omega \mathrm{cm}$. After a buffered HF dip to remove surface oxide, $100 \mathrm{~nm}$ of chrome was sputtered onto the front side, photoresist spun on, and the sample exposed with a chromeon-glass mask. The mask contained a number of different electrode sizes - from $6 \mathrm{~mm} \times 4 \mathrm{~mm}$ to $125 \mu \mathrm{m} \times 125 \mu \mathrm{m}$. The photoresist was developed, then the exposed chrome etched. Removal of the remaining photoresist was followed by a buffered HF etch and the samples were etched in TMAH, $25 \%$ solution at $60^{\circ} \mathrm{C}$, for $40 \mathrm{sec}$. Finally, the chrome was etched, and the samples rinsed and blowdried in $\mathrm{N}_{2}$.

A second set had 70nm-thick ITO layers deposited by sputtering, followed by photolithographic contact definition using the same mask as for set 1 . The ITO layer was etched using hydrochloric acid. Chrome-gold Ohmic contacts were provided on the back sides of all wafers by evaporation $(10 \mathrm{~nm} \mathrm{Cr}$, followed by $100 \mathrm{~nm} \mathrm{Au}$ ).

An asymmetric structure (n-type top electrodes (n-Si or ITO); p-type substrate), allows us to define two regimes in our MOS structure. Applying a negative potential to the top electrode allows higher currents to flow (negative bias); a positive potential results in lower currents (positive bias). In similar oxide based resistive systems, externally controlled current compliance has been required for successful operation. High current passing through thin oxide film could cause irreversible hard breakdown thus leading to device failure. An asymmetric design allows us to cycle the device without the need for external current compliance. 
I/V and I/t measurements were performed using a Keithley 4200 Semiconductor Characterisation System and a Signatone probe station. STM measurements were taken using a Nanosurf easyScan 2 Scanning Tunnelling Microscope. Atomic Force Microscopy (AFM) measurements were taken using a Veeco Dimension 3100 AFM operating in contact mode.

All experiments were performed in ambient conditions in an open laboratory. All shown results are preformed on the devices with the top ITO contact, $37 \mathrm{~nm}$ thick active layer and 11 at $\%$ excess $\mathrm{Si}$ unless otherwise specified. 


\section{Results and discussion}

\subsection{Current - voltage characteristics}

a)

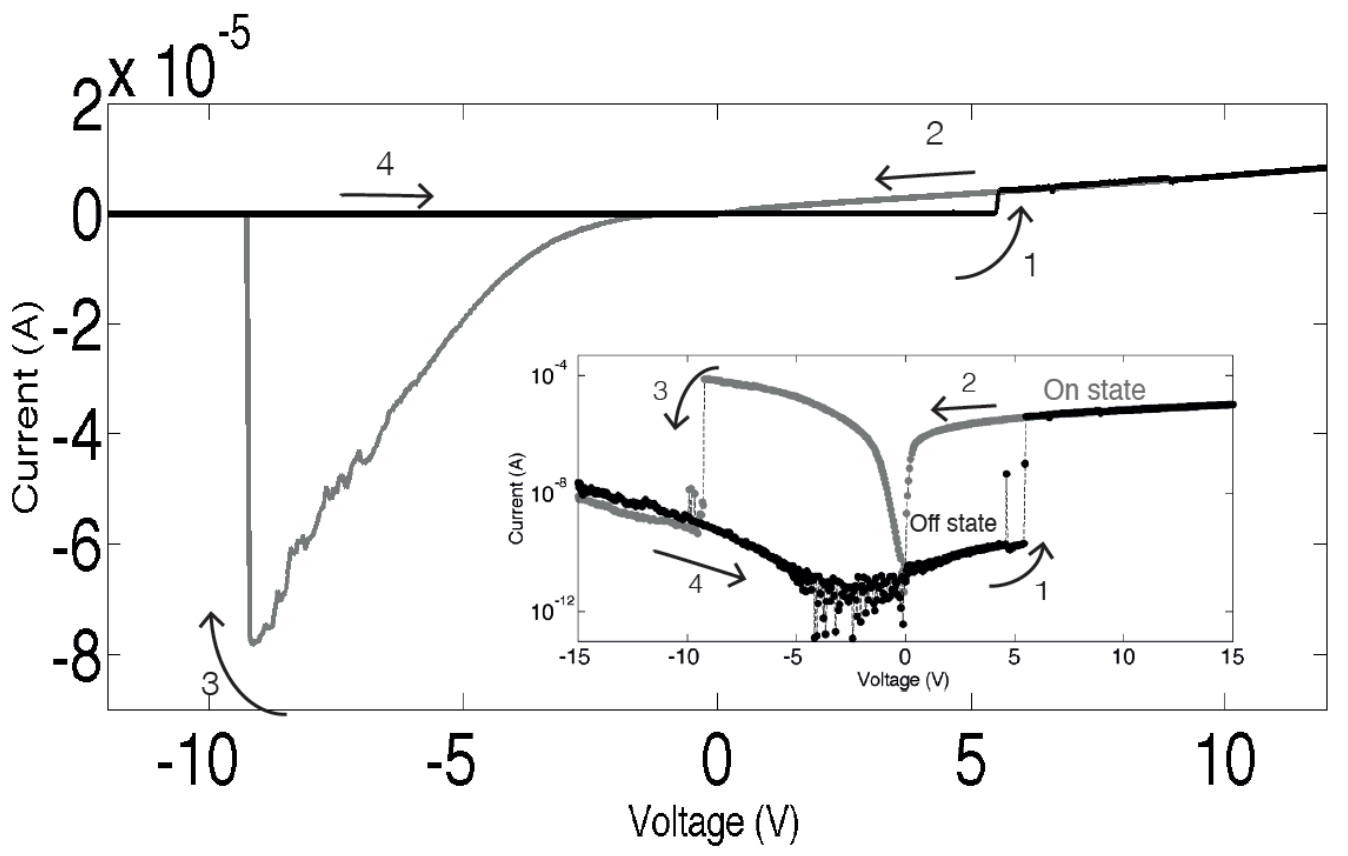

b)
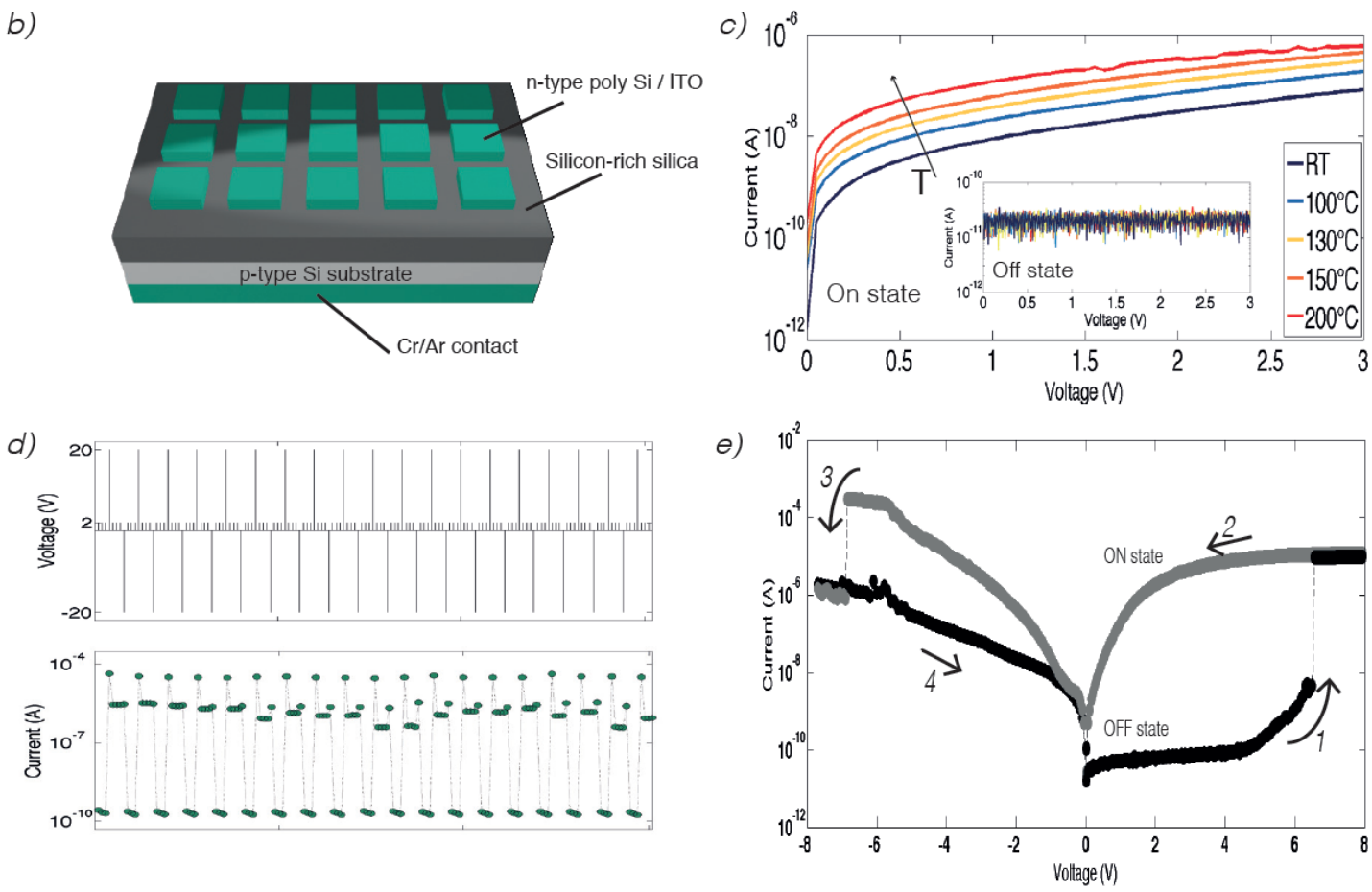

Figure 1. (colour online) (a) IV characteristics $(125 \mu \mathrm{m} \times 125 \mu \mathrm{m}$ top electrode). The black line in the positive bias shows a transition from OFF to ON state. The grey line shows a full ON state afterwards. The grey line in the negative bias shows a transition from ON to OFF state and the black line shows a full OFF state afterwards. Inset: Logarithmic representation. (b) Device schematic. (c) ON and OFF states dependence on increasing 
temperature. (d) Switching cycles using voltage pulses of $+20 \mathrm{~V},-20 \mathrm{~V}$ and $+2 \mathrm{~V}$ for setting, resetting and reading, respectively. (e) IV characteristics for device with poly-Si top contact (30at\% excess $\mathrm{Si}, 37 \mathrm{~nm}$ thick layer, $125 \mu \mathrm{m} \times 125 \mu \mathrm{m}$ contact size).

Figure 1(a) shows typical I/V results (contact size: $125 \mu \mathrm{m} \times 125 \mu \mathrm{m}$ ). Hysteresis is evident in both positive and negative bias, and the data show the pinched hysteresis curve characteristic of memristive systems. In positive bias, the initial high impedance (OFF) state switches to a low impedance (ON) state at a threshold voltage (black line in the positive bias). This is the "set" process. Reducing the voltage below threshold does not switch the device to its initial state, and much larger currents flow (grey line). Resistance contrast between the two states is up to six orders of magnitude in devices studied to date; four orders are typical. Transitions between states are abrupt (ie faster than the sampling time of our measurement) and do not depend on voltage sweep speed. The results shown are obtained with an acquisition time of around $15 \mathrm{~ms}$ per point (the maximum resolution of our equipment), which is equivalent to a sweep rate of $3.33 \mathrm{Vs}^{-1}$ with 300 points sampled. After switching, the device stays in the low impedance state until a negative bias is applied and certain critical current is reached. Although the low resistance to high resistance ("reset") switching process can be achieved in both polarities, the negative bias is much more efficient as higher currents can be achieved with lower voltages than in positive bias. A sharp drop in current can be seen (grey line in negative bias) when the required current level is reached under negative bias. Note in the example shown in figure 1a that the device returns to the initial high resistance OFF state following the reset process - in some cases it is possible to return the device to a partially OFF state with an intermediate resistance (see figure 2). The formation ("set") process can be achieved by again applying a positive bias. We note that the set process is also achievable under negative bias (Figure 2 inset), but its occurrence is less likely (and normally occurs only for larger contacts) than in the case of positive bias. 


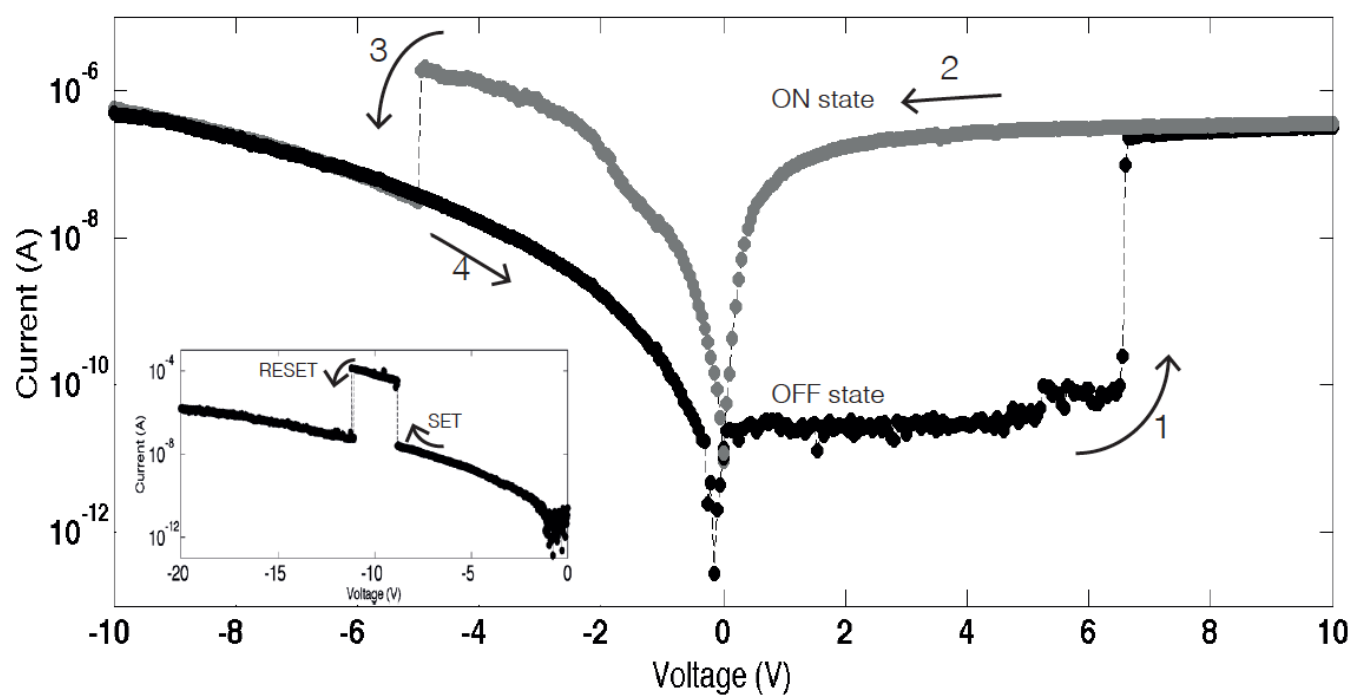

Figure 2. IV characteristics $(125 \mu \mathrm{m} \times 125 \mu \mathrm{m}$ top electrode). Inset: Unipolar switching for device with ITO contact $(250 \mu \mathrm{m} \times 250 \mu \mathrm{m}$ contact size $)$

Samples with silicon top contacts exhibited switching behaviour that was qualitatively similar to those with ITO contacts (Figure 1e), but higher reset currents were required, leading to reduced device endurance. We believe that this can be attributed to an additional annealing step at $950^{\circ} \mathrm{C}$ required to maximise the conductivity of the n-type poly-Si layer. This is likely to produce a high density of silicon nanoinclusions within the film as a result of phase separation, allowing strong conductive pathways to form. Future implementations of all-silicon memristors will therefore require careful control of the processing thermal budget.

Set and reset voltages can vary from the values shown in Figure 1a, depending on the history of the devices (duration and magnitude of the last applied voltage bias). However, short voltage pulses of $\pm 15 \mathrm{~V}$ or greater almost always trigger switching in healthy devices. These switching processes are repeatable, and the states stable for at least 120 hours at room temperature. Figure 1(d) illustrates sequential cycling between high and low resistance states (device programming). For the results shown in Figure 1(d) a programmed sweep mode was used with a sweep speed of around 50ms per point (the maximum resolution of our equipment in this mode). The applied voltages were: $+20 \mathrm{~V}$ (set), $-20 \mathrm{~V}$ (reset), and $+2 \mathrm{~V}$ (read). In the best devices programming can be achieved using $10 \mathrm{~V}$ pulses as short as 90ns (the limit of our equipment resolution - for these measurements a stand-alone 
pulse generator was used, rather than the Keithely 4200). Reset current in these devices can be up to $100 \mu \mathrm{A}$ (typical is around $10 \mu \mathrm{A}$, and in the best devices this can be as low as $2 \mu \mathrm{A}$ (Figure 2)), which is still sufficiently low for memory applications. The read operation uses approximately an order of magnitude less current in the ON state. The variations in reset current are likely an effect of nonhomogeneous film deposition. Material optimisation should provide better uniformity of reset currents and programming voltages.

Figures 1(c) shows conduction dependence on temperature in both ON and OFF state. Result shows typical semiconductor behaviour (resistance decreases with increased temperature), suggesting no metallic conduction in the ON state. Temperature increase does not affect the OFF state.

Figure 2 shows an IV curve for a sample with an ITO top contact in which the reset process puts the device into a partially OFF state; although the resistance is reduced by approximately one order of magnitude by the reset pule, the device does not return to the initial fully OFF state. This suggests the possibility of multi-level operation of devices.

The ON current does not show a clear tendency to scale with contact size (we tested this with the 3 contact sizes of $125 \mu \mathrm{m} \times 125 \mu \mathrm{m}, 250 \mu \mathrm{m} \times 250 \mu \mathrm{m}$ and $500 \mu \mathrm{m} \times 500 \mu \mathrm{m})$, suggesting carrier transport via individual conductive pathways. Contrary to other reports, in which switching is attributed to ionic diffusion from metallic contacts ${ }^{9}$, the switching in our device is an intrinsic property of the silicon-rich silica layer, so we hypothesise that the conductive pathways are related to the excess silicon content. In addition, we note that indium tin oxide is known to be very good diffusion barrier ${ }^{10}$, further supporting our conclusion that metallic diffusion is not the source of the resistive switching. Moreover, devices with poly-silicon contacts show very similar resistive switching behaviour (Figure 1(e)). When devices are heated up to $200^{\circ} \mathrm{C}$ during test, the conductivity in the $\mathrm{ON}$ state shows a reversible increase, confirming the non-metallic nature of the conduction path (Figure 1(c)). As the current is determined by the conductive pathway rather than the availability of carriers from the substrate, we conclude that the pathways are silicon, rather than diffused metal ions from the ITO top contact, or from contamination of the active layer. Contrary to this, systems exhibiting metallic filament conduction show a decrease in the ON state conduction with increasing temperature due to filament melting ${ }^{11}$. 
Also shown in Figure 2 (inset) is unipolar switching of the device under negative bias. Both set and reset processes can be seen, with reset occurring once the current has reached a critical value. The switching process is thus inherently unipolar.
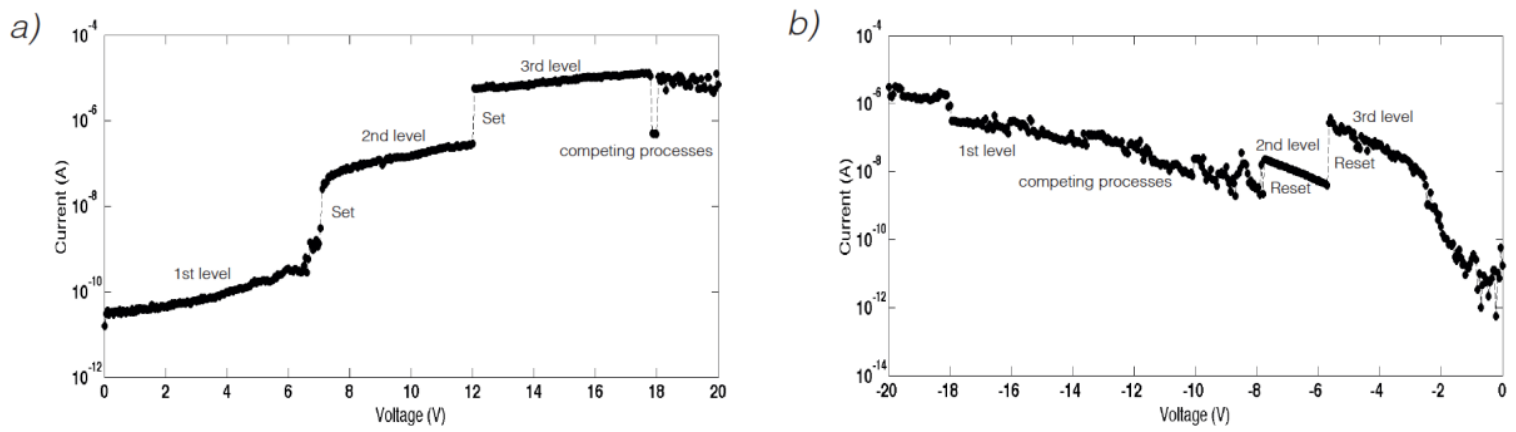

Figure 3. (a) IV curve (in positive bias) shows three distinct levels (two set processes and competing process) (b) IV curve (in negative bias) shows three distinct levels (two reset processes and competing process).

We note that the both ON read current and reset current could be increased if much larger contacts are used $(2 \mathrm{~mm} \times 2 \mathrm{~mm}$ or larger). In this case it is likely that a number of parallel conduction paths are formed. Further evidence of multi-level switching is shown in Figure 3. In this case, three distinct levels, together with competing set/reset processes are shown in Figure 3(a) and 3(b).

\subsection{Current - time characteristics}

Figures 4(a) and 4(b) show current-time graphs under constant voltage biases of $-8 \mathrm{~V}$ and $-10 \mathrm{~V}$ for the $2 \mathrm{~mm} \times 2 \mathrm{~mm}$ contact: it is evident that formation (set) and destruction (reset) processes act in opposition. In the case of larger contacts, it is likely that multiple conduction paths are formed, resulting in increased reset negative voltage and current. Clear transitions between two states can be seen with rapid current drops (up to 10\%) followed by slower, exponential, increases indicating multiple conduction paths where switching occurs in one or a few paths while other paths remains intact. Recovery speed is proportional to the applied voltage: the average time constant decreases from $10 \mathrm{sec}$ to around $0.5 \mathrm{sec}$ on changing the bias from $-8 \mathrm{~V}$ to $-10 \mathrm{~V}$. Higher negative voltages reset the device without repetitive transitions to the $\mathrm{ON}$ state. However, the $\mathrm{ON}$ state is stable if the bias is lower than 6V. We distinguish two conditions: high field and low current (positive bias) in which pathway formation is favoured, and high field and high current (negative bias) in which destruction 
dominates. We therefore propose a switching mechanism based on competing field-driven formation and current-driven destruction of conductive pathways.

a)

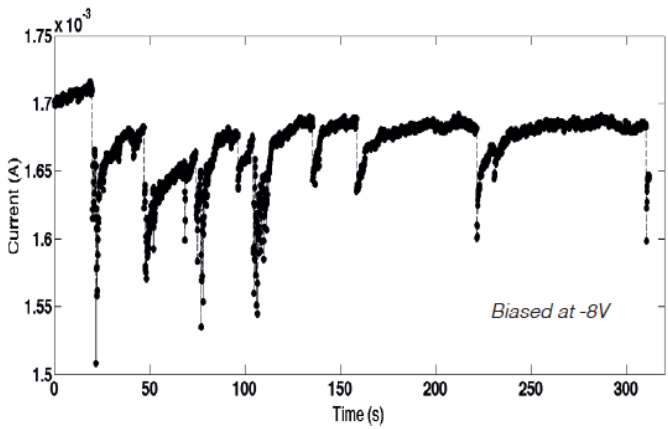

b)

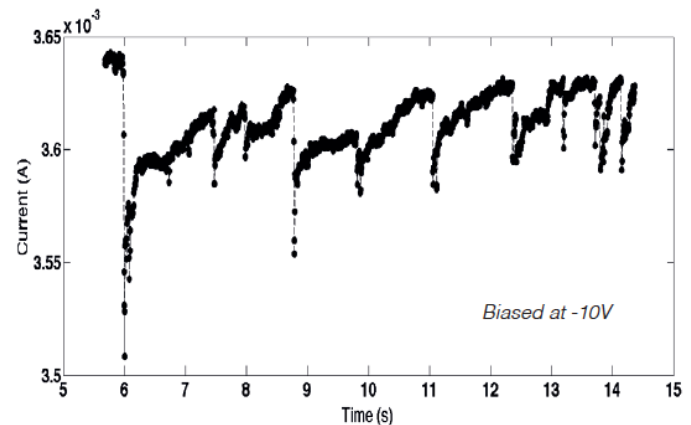

Figure 4. Current-time graphs under a constant voltage bias of (a) $-8 \mathrm{~V}$ and (b) $-10 \mathrm{~V}$.

\subsection{Impedance spectroscopy and conduction mechanism}

Impedance Spectroscopy ${ }^{1,12}$ reveals different conduction mechanisms in ON and OFF states, and is used to examine the nature of conducting path. In the OFF state, a single arc suggests an equivalent circuit model with a single parallel capacitor and resistor $\left(R=2.81 \times 10^{10} \Omega, C=2.61 \times 10^{-12} \mathrm{~F}\right)$. This high resistance is governed by the bulk properties of the $\mathrm{SiO}_{\mathrm{x}}$ layer. However, in the ON state, two clear arcs are seen, suggesting additional parallel resistances and capacitances appearing in series. This is consistent both with formation and destruction of conductive pathways, and with previous studies of carrier transport in such films. The latter can be modelled as shown in the inset of Figure $5 \mathrm{~b}$ $\left(\mathrm{R}_{1}=1.68 \times 10^{6} \Omega, \mathrm{C}_{1}=1.72 \times 10^{-10} \mathrm{~F} ; \mathrm{R}_{\mathrm{eq}}=2.58 \times 10^{6} \Omega, \mathrm{C}_{\mathrm{eq}}\right.$ (equivalent capacitance of series capacitors) $=3.03 \times 10^{-10} \mathrm{~F}$ ). The imaginary part of the impedance remains negative at frequencies between $1 \mathrm{~Hz}$ and $10^{7} \mathrm{~Hz}$, suggesting no inductive behaviour, which would be typical for metallic filaments. 
a)

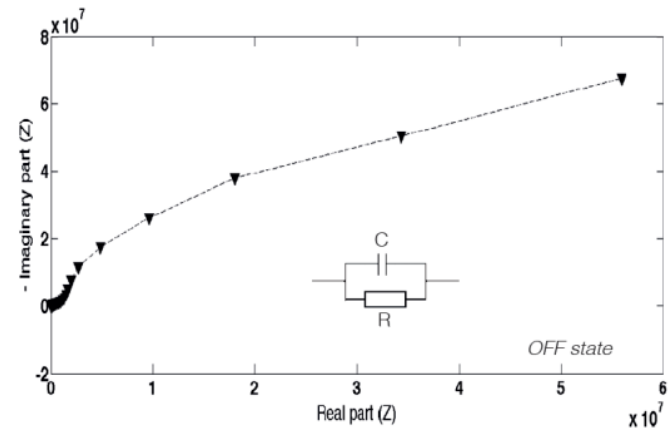

C)

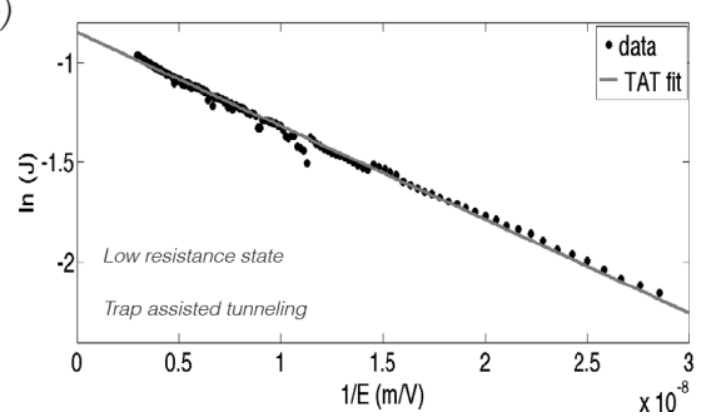

b)

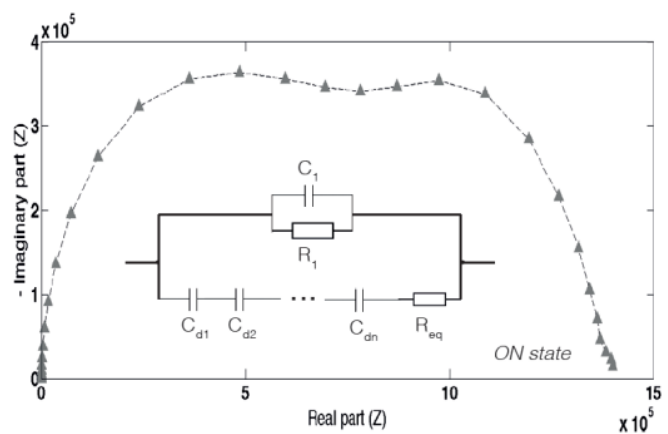

d)

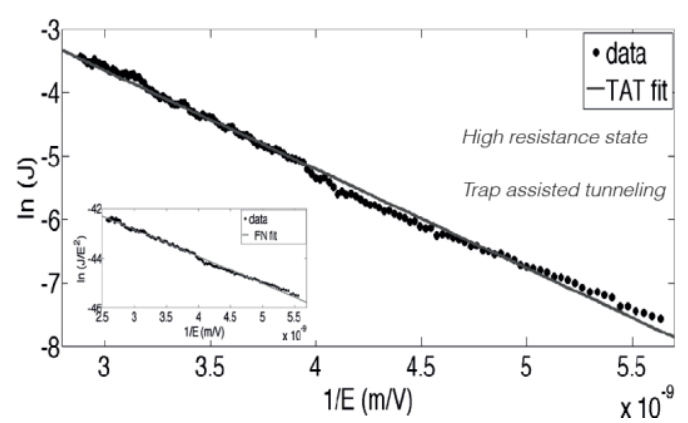

Figure 5. Cole - Cole plots with equivalent circuits under 1V in (a) OFF state and (b) ON state. Trap assisted tunnelling fit in (c) ON (low resistance) state and (d) OFF (high resistance) state (Inset: Fowler-Nordheim tunneling fit in OFF state).

The trap-assisted tunnelling dominates the conduction mechanism in both ON (low resistance) and partially OFF states (high resistance) (here, partially off refers to the black line in reverse bias in Figure 2; in the fully OFF state, there is insufficient current to measure conduction). Figure 5(d) shows data fit in the partially OFF state with the trap assisted tunneling model. The obtained trap depth is $0.52 \mathrm{eV}$. A reasonably good fit (especially at higher fields) can also be achieved with the Fowler-Nordheim tunnelling (F-N) model, as shown in the inset of Figure 5(d). In this case, a barrier height of $0.66 \mathrm{eV}$ is obtained which is consisted with literature results reported by DiMaria ${ }^{13}$.

ON state conduction is modelled best using trap assisted tunnelling for all applied fields (Figure 5(c)). The obtained value for trap depth is $0.086 \mathrm{eV}$. In order to make sense of this result, we note that it is likely that the matrix in between the silicon nanoinclusions will be a suboxide of silicon with a much smaller band gap than stoichiometric $\mathrm{SiO}_{2}$, resulting in a much lower barrier height - intermediate between the band offset of the $\mathrm{Si} / \mathrm{SiO}_{2}$ interface, and zero. A chain of nanoparticles is formed, and transport proceeds via trap-assisted tunnelling (TAT) between adjacent nanoparticles ${ }^{14,15}$. The larger trap depth in the partially OFF state can be ascribed to a reduction in the stoichiometry of gaps in the 
conductive pathway following removal of nanoinclusions by Joule heating. The trap depth here increases, moving towards a value close to the $\mathrm{Si} / \mathrm{SiO}_{2}$ band offset. Note that we did not observe Fowler-Nordheim tunnelling in devices in the fully ON state.

\subsection{Scanning Tunnelling Microscopy \& Atomic Force Microscopy}
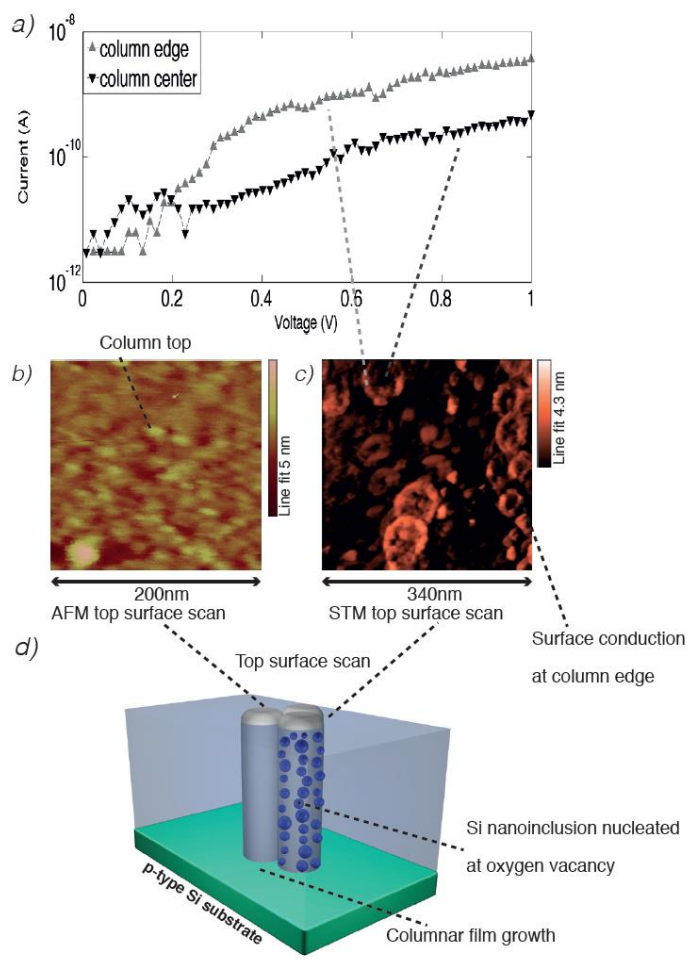

Figure 6. (colour online) (a) STM I/V curves for the edge point (b) Atomic force microscopy scan of the surface top side showing surface features attributed to the tops of growth columns (c) Scanning tunnelling microscopy scan of a sample surface (different area to that in b)) showing enhanced conductivity at column edges (d) Schematic of columnar structure of switching film and switchable site.

It is well established that films grown by sputtering often exhibit columnar or granular growth ${ }^{16}$. Boundaries between adjacent columns can extend through the whole thickness of the film, effectively connecting the top and bottom of the active layer. Atomic Force Microscopy (Figure 6(b)) of sample surfaces indicated periodic 5-10nm high circular dome-shaped surface features that varied in diameter from $10 \mathrm{~nm}$ to $50 \mathrm{~nm}$. STM of these structures showed high conductivity at the edges of the features, and low conductivity at the centre (Figure 6(c)). Such pathways are around 5-30nm in diameter, suggesting that devices may be scaled down to the nanometre dimensions to achieve very high levels 
of integration. Figure 6(a) shows I/V measurements of the two regions, highlighting the difference in conductivity. Figure 6(d) is a schematic of the film structure, showing columnar structures with silicon nanoinclusions nucleating at column boundaries. Such a structure is consistent with both AFM and STM results.

\subsection{Switching model}

In order to explain the memristive behaviour of our devices, we first note that silicon-rich silica is a metastable material that readily segregates into silicon and silicon dioxide ${ }^{17,}{ }^{18}$. It contains a high concentration of oxygen vacancies ${ }^{19}$, which can be driven by high temperature annealing and consequent diffusion of vacancies and silicon to form silicon nanoclusters. In the initial stages of annealing, sub-nanometre clusters nucleate at oxygen vacancies ${ }^{20}$, increasing in number and growing by Ostwald ripening at longer times and/or higher temperatures as silicon diffuses. We propose that voltages applied across our devices drive oxygen vacancy migration, producing a field-driven phase separation of the active layer ${ }^{21}$. This separation is enhanced by structural defects such as nano-scale cracks or inclusions ${ }^{18}$; significantly, films grown by sputtering typically exhibit columnar or granular growth $^{16}$; boundaries between adjacent growth columns constitute structural defects that can nucleate phase separation. The rate of nucleation, as well as the number density and size of silicon nanoinclusions produced by the migration of oxygen vacancies, will increase with applied field until a critical point at which percolation pathways can be formed along the column boundaries (corresponding to the "set" process). Current transport in devices in the ON state is by trap assisted tunnelling, suggesting that conductive pathways through our material are not continuous filaments, but instead a sequence of separate but neighbouring silicon nanoinclusions (analogous to aggregated oxygen vacancies). The low activation energy in ON state (found for the TAT fit) is typical for conduction through oxygen vacancy defects. Such a low value is understandable if we assume that transport is through a semi-continuous array of adjacent silicon nanoinclusions in a sub-oxide matrix.

The stoichiometry of the inter-inclusion matrix varies with applied field and Joule heating, with a more stoichiometric matrix (in the case of a partially OFF device) yielding a higher barrier height than for a very Si-rich matrix. 
The switching process is intrinsically unipolar (Figure 2), though we employ bipolar operation as this enables more stable programming.

The proposed process is shown schematically in Figure 7.

a)

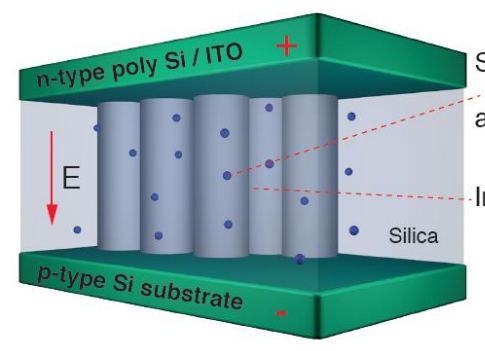

d)

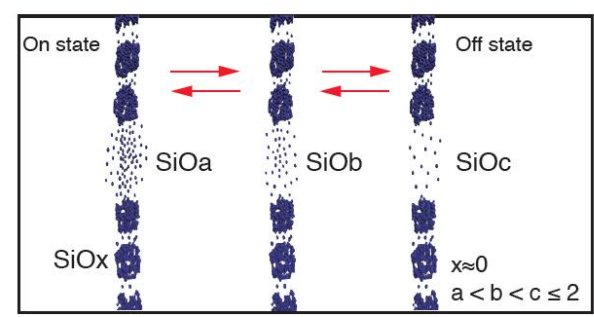

b) Si nanoinclusion nucleated at oxygen vacancy Internal columns \& boundaries
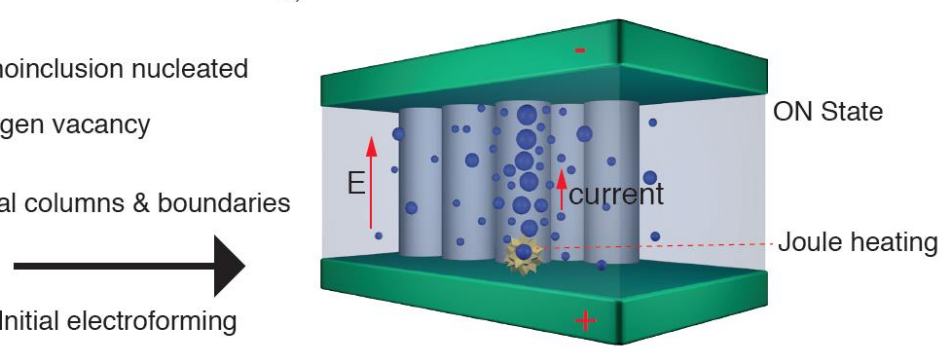
Annihilation $\downarrow \longleftarrow$ Recovery

c)

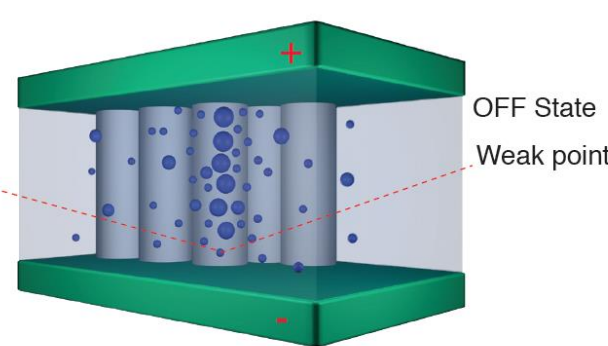

Figure 7. Schematic of one cycle process. (a) Initial OFF state before applying the electric field, showing asgrown silicon nanoinclusions nucleated at oxygen vacancy sites. (b) ON state after the chain formation, showing extra silicon nanoinclusions produced by field-driven migration of oxygen vacancies. (c) Annihilation process due to Joule heating. (d) Silicon and oxygen distribution at the weak point.

Although Joule heating is the main driving force for the reset process, the electric field undoubtedly plays an important role. It is known that the silicon/silica interface is more favourable for the formation of oxygen vacancies ${ }^{22}$ and thus more porous. Consequently, it is more likely that the position of the weak (switching) point is near the substrate/silica interface. As the oxygen vacancies behave as positively charged species, vacancies would be pushed towards the substrate during the set process (positive bias), recovering the broken path. In the reset process (negative bias) energy from the high local Joule heating overcomes the binding energy of vacancies, and the electric field consequently pushes them towards the top electrode, rapturing the conduction path. 


\section{Conclusion}

In conclusion, we report a study of resistive switching in silicon-rich silicon dioxide films grown by co-sputtering. Our results suggest that conductive pathways are formed at grain boundaries by the field-driven nucleation of oxygen vacancies. Such pathways are not continuous, but are chains of silicon nanoinclusions separated by a highly sub-stoichiometric oxide matrix. A combination of applied field and Joule heating controls the distribution of oxygen vacancies, which is reflected in changes in the device resistance of four orders of magnitude or more. Our memristive RRAM device differs from those previously reported by not requiring diffusing metallic contacts ${ }^{5}$, and not being limited by surface conduction to vacuum operation ${ }^{4}$. The resistive states are stable, switching pulses can be $90 \mathrm{~ns}$ or shorter, and switching currents are around $10 \mu \mathrm{A}$. Such devices are very promising for application in memristive systems such as high-density semiconductor memories. We note that further investigation (underway) should yield more detailed insight into the microscopic switching mechanism.

\section{References}

1. P. Heremans, G. H. Gelinck, R. Muller, K. J. Baeg, D. Y. Kim and Y. Y. Noh, Chemistry of Materials 23 (3), 341-358 (2011).

2. L. O. Chua, IEEE Transactions on Circuit Theory CT18 (5), 507-\& (1971).

3. D. B. Strukov, G. S. Snider, D. R. Stewart and R. S. Williams, Nature 453 (7191), 80-83 (2008).

4. J. Yao, Z. Z. Sun, L. Zhong, D. Natelson and J. M. Tour, Nano Letters 10 (10), 4105-4110 (2010).

5. S. H. Jo, K. H. Kim and W. Lu, Nano Letters 9 (1), 496-500 (2009).

6. A. Sinitskii and J. M. Tour, ACS Nano 3 (9), 2760-2766 (2009).

7. M.-J. Lee, C. B. Lee, D. Lee, S. R. Lee, M. Chang, J. H. Hur, Y.-B. Kim, C.-J. Kim, D. H. Seo, S. Seo, U.-I. Chung, I.-K. Yoo and K. Kim, Nature Materials advance online publication (2011).

8. S. H. Jo and W. Lu, Nano Letters 8 (2), 392-397 (2008).

9. R. Huang, L. J. Zhang, D. J. Gao, Y. Pan, S. Q. Qin, P. R. Tang, Y. M. Cai and Y. Y. Wang, Applied Physics a-Materials Science \& Processing 102 (4), 927-931 (2011).

10. C. M. Liu, W. L. Liu, W. J. Chen, S. H. Hsieh, T. K. Tsai and L. C. Yang, Journal of the Electrochemical Society 152 (3), G234-G239 (2005).

11. F. El Kamel, P. Gonon, C. Vallee, V. Jousseaume and H. Grampeix, Applied Physics Letters 98 (2), 023504 (2011). 
12. E. Barsoukov, MacDonald, J. Ross, Impedance Spectroscopy: Theory, Experiment, and Applications. (John Wiley \& Sons, Inc., Hoboken, New Jersey, 2005).

13. D. J. Dimaria, D. W. Dong, C. Falcony, T. N. Theis, J. R. Kirtley, J. C. Tsang, D. R. Young, F. L. Pesavento and S. D. Brorson, Journal of Applied Physics 54 (10), 5801-5827 (1983).

14. M. Porti, M. Avidano, M. Nafria, X. Aymerich, J. Carreras and B. Garrido, Journal of Applied Physics 98 (5), 056101 (2005).

15. O. Jambois, Y. Berencen, K. Hijazi, M. Wojdak, A. J. Kenyon, F. Gourbilleau, R. Rizk and B. Garrido, Journal of Applied Physics 106 (6), 063526 (2009).

16. M. T. P. McCann, D. A. Mooney, M. Rahman, D. P. Dowling and J. M. D. MacElroy, Acs Applied Materials \& Interfaces 3 (2), 252-260 (2011).

17. L. A. Nesbit, Applied Physics Letters 46 (1), 38-40 (1985).

18. G. A. Kachurin, K. S. Zhuravlev, N. A. Pazdnikov, A. F. Leier, I. E. Tyschenko, V. A. Volodin, W. Skorupa and R. A. Yankov, Nuclear Instruments \& Methods in Physics Research Section B-Beam Interactions with Materials and Atoms 127, 583-586 (1997).

19. S. Cueff, C. Labbe, B. Dierre, F. Fabbri, T. Sekiguchi, X. Portier and R. Rizk, Journal of Applied Physics 108 (11), 113504 (2010).

20. C. R. Mokry, P. J. Simpson and A. P. Knights, Journal of Applied Physics 105 (11), 114301 (2009).

21. A. Sarikov, V. Litovchenko, I. Lisovskyy, I. Maidanchuk and S. Zlobin, Applied Physics Letters 91 (13), 133109 (2007).

22. A. X. Chu and W. B. Fowler, Physical Review B 41 (8), 5061-5066 (1990).

\section{Acknowledgements}

We acknowledge the valuable assistance provided by Steve Etienne (cleanroom processing). AM was supported by a UCL Overseas Research Scholarship. Funding was provided by EPSRC. 